\title{
Esbozo del Humanismo español
}

\section{Francisco Rico}

\section{(2) OpenEdition}

\section{Journals}

Edición electrónica

URL: http://journals.openedition.org/studifrancesi/9399

DOI: 10.4000/studifrancesi.9399

ISSN: 2421-5856

\section{Editor}

Rosenberg \& Sellier

\section{Edición impresa}

Fecha de publicación: 1 diciembre 2007

Paginación: 526-531

ISSN: 0039-2944

\section{Referencia electrónica}

Francisco Rico, «Esbozo del Humanismo español», Studi Francesi [Online], 153 (LI | III) | 2007, online dal 30 novembre 2015, consultato il 12 janvier 2021. URL: http://journals.openedition.org/ studifrancesi/9399; DOI: https://doi.org/10.4000/studifrancesi.9399

\section{(c) (i) (2) $\Theta$}

Studi Francesi è distribuita con Licenza Creative Commons Attribuzione - Non commerciale - Non opere derivate 4.0 Internazionale. 


\section{Esbozo del Humanismo español}

Tout au long $d u X V^{e}$ siècle, le monvement de renouveau culturel inauguré par Pétrarque se fit progressivement sentir dans la Péninsule ibérique à travers la curiosité de quelques intellectuels et le snobisme de quelques grands seigneurs. L'ouvre et l'exemple de Nebrija, sous les Rois Catholiques, lui donnèrent l'impulsion nécessaire pour se développer à l'époque des Habsbourgs et pour fournir de solides contributions à la philologie et à d'autres domaines de la culture de haut niveau. Par ailleurs, l'enseignement de base, amplement répandu au XVI siècle, n'a jamais accueilli les présupposés de l'Humanisme avec trop de rigueur. Grace à cela, le classicisme n'atteignit jamais l'bégémonie absolue qui fut sienne dans d'autres pays; dès lors les écrivains disposèrent d'une plus grande liberté dont le fruit le plus important fut la création d'un genre fondamental de la modernité littéraire: le roman réaliste.

En la primera mitad del siglo Xv, bastantes españoles, principalmente magnates, miembros del alto clero y burócratas, se dejaron fascinar por la revolución que se había producido en Italia. Los viajes, los viajeros, algunos libros les pusieron ante los ojos la maravilla de una resurrección de la Antigüedad que estaba transfigurando el pensamiento, las artes, la misma vida cotidiana de las gentes refinadas.

En general, los precursores cuatrocentistas - Bernat Metge, Juan de Mena, el Marqués de Santillana, Juan de Lucena, digamos - veían sólo los resultados más epidérmicos de los studia bumanitatis y a menudo aspiraban a emularlos con los medios que tenian a mano y sin variar la formación que les era propia (y Boccaccio les sirvió muchas veces de puente hacia la Antigüedad y modelo de proceder). ¿Había que multiplicar las pinceladas mitológicas, las alusiones a la historia y a la geografía del mundo grecolatino? Pues allí estaban el Catolicón, las Etimologías y el De imagine mundi, tan ricos en datos, mejores o peores, a tal propósito. ¿El ideal del estilo era la elocuencia latina? Pues ellos se sabían al dedillo las recetas de los dictatores, y muchos, además, las del leguleyo a la escolástica.

Esos esfuerzos tempranos produjeron algunos libros estimables como literatura y estimularon un cierto clima favorable en las cortes y en otros ambientes, pero no bastaban para cambiar demasiado el panorama. Juristas mayormente, los autores se habían criado a pechos de los autores medievales, y cuando olfatearon los sugestivos caminos que arrancaban de Italia llevaban ya irremediables vicios de origen. «Yo fui a Roma grandevo», confiesa uno de ellos. A todos se aplica cosa similar. Llegaron tarde a la cultura cuyo paradigma brindaba Italia, y hubieron de quedarse en amateurs, a menudo sin entenderla plenamente ni asimilarse sino un chillón barniz de clasicismo. Pero los studia bumanitatis, antes que nada, eran una paideía, una institutio o educación desde los fundamentos; y, sin rehacer los fundamentos, sólo cabía dar palos de ciego.

A la instauración de una pedagogia basada en el Humanismo contribuyeron decisivamente los grandes nobles, a quienes la gestación del estado moderno forzaba a reorientar sus energías. Por naturaleza y por historia, las abstracciones del escolas- 
ticismo nada decían a la mentalidad y modo de vida aristocráticos. La concreción y el ámbito de evocaciones de los studia bumanitatis, en cambio, podían sonarles no poco atractivos. Al principio, por otro lado, ni siquiera era imprescindible hacerse demasiado cargo de su contenido: bastaba que se tratara de una orientación nueva y distinta para convenir a las exigencias de los magnates.

Con frecuencia se han malentendido los términos de la cuestión y argumentado, por ejemplo, que difícilmente cabe atribuir a Alfonso el Magnanimo, el cardenal Pedro Gonzalez de Mendoza, Isabel la Católica o tal o cual otro mecenas un lugar de relieve en la diseminación del Humanismo, porque nunca llegó a dominar personalmente los textos clásicos, ni menos los métodos de la filología. Es una opinión absurda. A nadie se le ocurriría poner en tela de juicio que el filántropo que financia unas determinadas investigaciones hace un aporte valioso al avance de la materia en cuestión: no se requiere ser biólogo para advertir que la biología es hoy una disciplina central en el dominio del saber y para apoyarla en consecuencia.

Conviene no perder de vista tan elementales consideraciones, para entender a derechas cómo fue consolidándose el Humanismo: el modelo propugnado por los humanistas no llegó a instaurarse en la importante proporción en que lo hizo porque tuviera estas o aquellas virtudes intrínsecas, que sin duda las tenía, aunque no forzosamente mejores que el modelo rival de la escolástica, sino porque ganó un prestigio singular y se plasmó en unos modos de vida que las clases superiores, a veces por convicción, más comúnmente por intuición y ganas de estar à la page, tendieron a adoptar con entusiasmo.

Entre 1480 y 1520, el Humanismo se afianza en España a un paso increíblemente rápido. Tanta es la celeridad del cambio, tan ansiosamente se van quemando etapas, que las metas de un día resultan al siguiente desplazadas por otras.

La parte constitutiva del proceso ocurre toda durante la vida y en buena medida gracias a la obra de Antonio de Nebrija. Los cimientos los puso Nebrija en 1481 publicando unas Introductiones latinae que se proponían, sencillamente, enseñar el buen latín de Cicerón, con la perspectiva de Valla y con el método de Guarino. Menos de quince años después, y adelantándose a Erasmo en la intención, Nebrija se decía cansado de otros trabajos y anunciaba el propósito de dedicar el resto de sus días a la filología bíblica trilingüe. A su muerte, en 1521, solo cuarenta años después de las Introductiones, el primer Humanismo español contaba con un monumento de la extraordinaria categoría de la Biblia Poliglota Complutense o, por alegar sólo otro nombre, con algunos de los más valientes libros de quien nunca negó su condición de discípulo y admirador de Nebrija: Juan Luis Vives.

Las escuetas Introductiones de 1481 fueron creciendo hasta convertirse en una verdadera enciclopedia lingüística, al tiempo que su autor proseguía las investigaciones sobre la fonética histórica del latín y del griego - sin parangón en su tiempo por la hondura y clarividencia - y las glosas jurídicas, médicas y bíblicas con las cuales aspiraba a compilar un gigantesco vocabulario de todos los saberes. El sentido unitario de su inmensa actividad se hace patente con especial transparencia y rotundidad en 1488, en la edición de las Introductiones en la que la versión castellana corre paralela al texto original.

«Para el colmo de nuestra felicidad y complimiento de todos los bienes», proclama ahí Nebrija, «ninguna otra cosa nos falta sino el conocimiento de la lengua», del buen latín. Él, en efecto, es el fundamento de «nuestra religión y república cristiana»: sin buen latín, los teólogos y los biblistas no tienen acceso a la obra de los Padres de la Iglesia, cuyo estilo y manera de argumentar son resueltamente clásicos; y sin los Padres de la Iglesia, los doctorcillos modernos no pueden beber en las aguas de la Escritura y han de quedarse en la ciénaga de los medievales que emplearon el galimatías 
escolástico que ellos han aprendido. El buen latín es asimismo la base del derecho, gracias a cuyo recto uso existe la civilización y «los hombres viven igualmente [= aeque] en esta gran compañía que llamamos ciudad». Como es base de la medicina, «por la cual se contiene nuestra salud y vida».

Pero ¿qué ocurre en España? Que, por no saber latín, los supuestos expertos en derecho y en medicina interpretan mal las fuentes de información que poseen y se ven privados de otras importantísimas; y si los juristas caen en los más grotescos errores por incomprensión y falta de sentido histórico en la lectura de los códigos («non habita ratione temporum»), los medicastros - se reía Nebrija con sus alumnos - llegan a confundir la uvula con la vulva...

Idéntico «laberinto de confusión» es el panorama de las restantes disciplinas: por ignorancia del latín, «todos los libros en que están escritas las artes dignas de todo hombre libre yacen en tinieblas sepultados» desde hace muchos siglos, «no menos que todas las otras buenas artes», y en particular «las artes que dicen de bumanidad, porque son propias del hombre en cuanto hombre». En suma: sin dominar cabalmente el latín, no hay medio de edificar una «ciudad» verdaderamente humana. La plenitud del individuo y de la comunidad empieza con un modesto manual de latín.

Es ése el programa máximo del Humanismo: la conquista de una nueva civilización, en todos los aspectos, gracias a la reconquista de las letras antiguas, entendiendo el latín como camino a la concreción, a la elegancia y la pertinencia de pensamiento, a la aprehensión de la realidad en tanto proceso histórico. Es el ideal que inspira la obra de los nombres mayores del movimiento, de Petrarca a Erasmo (y, para Nebrija, sobre todo de Valla), y que los menores fueron inevitablemente recortando: en los casos más lúcidos porque eran conscientes de que los medios no bastaban para alcanzar el fin. Yo lo he llamado "el sueño del Humanismo", y Anthony Grafton ha resumido felizmente en una sola frase el destino de esa ilusión: "the preservation of classical Latinity proved incompatible with the creation of a new intellectual world".

Nebrija vino a traer exactamente lo que más se necesitaba para una saludable implantación del Humanismo en la Península: unos ideales concretados en un método y apoyados en un modelo personal, un modelo de vida. Las Introductiones, reeditadas y revisadas continuamente, aportaron el método; y el prestigio del autor reforzó los ideales y dio el modelo que había de entusiasmar a multitud de ánimos jóvenes, desde modestos maestros de escuela hasta grandes eruditos.

Hasta el último tercio del siglo xv, los encuentros de los españoles con los studia bumanitatis no habían sido fecundos - cuando lo fueron - sino a escala individual, o si acaso en cenáculos limitados. El Humanismo sólo podía tener repercusión profunda si su núcleo constitutivo se ofrecía con la adecuada formulación didáctica y en el aula de enseñanza. El cambio substancial, capaz de renovar desde las raíces el conjunto de la cultura, requería algo distinto que las sugerencias de creadores o ensayistas italianos y que los experimentos singulares de curiales o magnates ibéricos: pedía libros de texto, repertorios de consulta, lecturas accesibles. Los principios expuestos a trazos sueltos, sin sistema, y las brillantes aportaciones aisladas debían reducirse a códigos escolares, sujetarse a normas.

La contribución de Nebrija llegó, además, en el momento justo, cuando el advenimiento de la imprenta impulsaba la difusión y la uniformidad de los materiales pedagógicos, y cuando la articulación de la Europa moderna, señaladamente en la España de los Reyes Católicos, ofrecía generosas oportunidades de promoción social a quienes recibieran la educación adecuada. Pero el Nebrisense tampoco estaba solo. Como preceptores en la corte regia o en los palacios señoriales, llegaron a finales del siglo xv Lucio Marineo Siculo, Pedro Mártir de Anglería, Alessandro y Antonio 
Geraldini y otros sólidos humanistas, cuya presencia asimismo se hizo sentir, más o menos duraderamente, en la universidad.

Esos estudiosos venían acompañados por una aureola que tenía que ver más con la moda que con el contenido de sus lecciones. Así, cuenta Pedro Mártir que cuando llegó a Salamanca, en 1488, el mero anuncio de que "un extranjero" iba a dar una conferencia pública reunió tal concurrencia, que "muchos tuvieron que ser sacados del aula medio asfixiados”, y la disertación, que versaba sobre Juvenal, tuvo tanto éxito, que el orador fue acompañado hasta su casa "como un vencedor desde el Olimpo”. ¿Hemos de suponer que todos los oyentes eran filólogos avezados, y tan seducidos por la poesía latina como para seguir "con oídos atentos" durante dos horas una docta elucidación de la sátira segunda? En absoluto. Los más serían sencillamente simpatizantes del Humanismo en un sentido amplio y vago, como cultura moderna y prometedora, cuyos grandes rasgos se conocen y se aprueban, incluso hasta la exaltación, pero cuyo escrutinio y cultivo se deja a los especialistas.

Las Introductiones y el ejemplo de Nebrija, la receptividad de las clases dirigentes y el fervor juvenil por las novedades, al arrimo de una favorable coyuntura social (y no sin las lógicas resistencias), afianzaron los studia bumanitatis tanto en la alta cultura y en la universidad como en la cultura general y en la enseñanza de base.

Importa no confundir ambos niveles. La historia del Humanismo se escribe hoy principalmente como historia de la alta cultura y, sobre todo, de la filología clásica. Es comprensible, porque, desvanecido el sueño grandioso e imposible de los pioneros, los resultados más perdurables del movimiento se dieron en ese dominio. Pero, precisamente al restringirse a la filología clásica, el Humanismo dejó de ser el poderoso fermento cultural que había sido, para quedarse, hasta hoy, en una mera disciplina especializada. Una disciplina relevante y productiva, notablemente como auxiliar de la historia, pero en definitiva marginal y cada vez más caracterizada (para decirlo en los términos de la sociología del conocimiento) como una "tribu académica".

Otro es el panorama si el Humanismo se contempla atendiendo a su penetración en la enseñanza de base y, a través de ella, en la cultura general. La repercusión de las técnicas filológicas es reducida, mientras la educación que se recibe en la escuela se extiende a vastas parcelas de la sociedad y, en particular, se hace presente en la literatura. Es en ese terreno donde el Humanismo produjo en España mejores rendimientos.

Con posterioridad a Nebrija, el supremo exponente español de unos studia bumanitatis concebidos como savia vital de todo conocimiento fue sin duda Juan Luis Vives (1492-1540). "Las lenguas - cree el valenciano - son las puertas de todas las disciplinas y artes", y hay que apropiárselas con la máxima exigencia filológica. "Sed meminerint homines studiosi - añade -, si nihil adiecerint linguis, ad fores tantum pervenisse eos artium, et ante illas, aut certe in vestibulo versari, nec plus esse Latine ac Graece scire quam Gallice et Hispanice, uso dempto, qui ex linguis eruditis potest accedere, nec linguas omnes labore illo propter se ipsas dignas esse, hoc est, si aliud nihil quaeratur, quippe propter exteriorem utilitatem tantummodo parantur..." Por ahí, la gigantesca obra de Vives parte del estudio del latín y del griego y con ellos se alza a la filosofía de la cultura y de la historia, a la psicología y a la epistemología, a la meditación sobre la ética y la política, a menudo en coincidencia y en alianza con Erasmo. Como Séneca, como Petrarca, Vives entiende la filología como un preámbulo: ineludible, pero sólo para progresar hacia horizontes mayores.

Vives aparte (y por encima), en la España de los Habsburgo el Humanismo técnico, profesional, tuvo un número no despreciable de cultores de primera categoría. Hernán Núñez de Guzmán (Pintianus, en el aparato de las ediciones críticas) restituyó o examinó magistralmente muchos lugares de Plinio y Mela (1544). Las 
ediciones de Varrón y Festo y las investigaciones sobre el derecho, la epigrafía o la numismática de Roma situaron a Antonio Agustín (1517-1586) entre las luminarias de su época. El padre Juan Luis de la Cerda dio a la luz un Virgilio exhaustivamente glosado (1609-1617) que gozó desde el primer momento de gran autoridad. La fama de las curiosísimas Disquisitiones magicae (1599-1600) de Martín Alonso del Río tiende a que se releguen a un segundo plano, injustamente, sus respetables estudios sobre la tragedia latina. Las versiones de Aristóteles y de sus escoliastas por Juan Ginés de Sepúlveda (1490-1573) combinaron la exactitud y la elegancia hasta un extremo antes no alcanzado. El De naturae philosophia, seu de Platonis et Aristotelis consensione (1554), y los comentarios a Platón de Sebastián Fox Morcillo fueron un firme punto de referencia en el Renacimiento tardío. Pedro de Valencia publicó una espléndida historia del escepticismo antiguo: Academica, sive de iudicio erga verum ex ipsis primis fontibus (1596). La erudición trilingue, la honda espiritualidad y las dotes poéticas de Benito Arias Montano (1527-1598) confluyeron en la monumental Biblia Poliglota de Amberes, que por deseo expreso de Felipe II vino a actualizar la empresa que en Alcalá de Henares habia impulsado el Cardenal Cisneros medio siglo atrás. Inmensa fortuna ha tenido durante siglos la Minerva sive de causis linguae latinae $\left(1562,1587^{2}\right)$ con la que Francisco Sanchez de las Brozas, el Brocense, revolucionó la gramática para defender la ratio frente al usus divinizado por Lorenzo Valla.

Basten esos nombres para confirmar que la filología humanística, hasta bien entrado el siglo XVII, tuvo en España una trayectoria no demasiado productiva, pero decorosa, perfectamente equiparable a los patrones de calidad del resto de Europa. La insistencia con que, desde Nebrija, se orientó al estudio de la Biblia hizo que en la Península se la contemplara a menudo con más prevención que en otras partes, pero ésa es también una de sus notas originales.

Cosa parecida puede decirse del otro sector de la alta cultura más favorecido en las historias del Humanismo. La antología, por otro lado tan sugestiva, de Perosa y Sparrow Renaissance Latin Verse incluye una única muestra de la poesía latina del Quinientos español: una oda de Garcilaso de la Vega. No echemos la culpa a los concienzudos antólogos, sino a la información de que disponían. La lírica y la épica hispanolatinas de los siglos XV y XVI fueron publicándose en volúmenes independientes y nunca se recogieron en las series de delitiae que en el Seiscientos se estamparon en Frankfurt, Amsterdam, Leyden. La consecuencia fue que en esa época de toma de conciencia y formación de la historiografía literaria se tuvo la impresión de que el Renacimiento no había producido en España poesía latina. Pero la verdad es que hubo entonces una producción notable en cantidad y dignidad, desde los madrigales amorosos de Hernán Ruiz de Villegas hasta la gran lírica devota de Arias Montano o los epigramas conceptuosos de Jaime Falcó.

La impronta mayor y más significativa del Humanismo en España no debe buscarse en las cumbres de la filología o la poesía latina, más o menos elevadas pero similares en toda Europa, sino en el ámbito de la educación general de base. Desde los días de los Reyes Católicos, las escuelas proliferaron extraordinariamente, y el núcleo de la mayoría de ellas estaba constituido por el aprendizaje del latín con una variable acogida de la literatura antigua. Esa proliferación tuvo un anverso y un reverso. El anverso consistió en que una proporción considerable de la población adquirió los rudimentos de la cultura clásica; el reverso estuvo en que el nivel de la enseñanza fue notoriamente bajo.

En efecto, en 1600 el numero de centros, municipales, catedralicios o filantrópicos, incluso en pequeños lugares, se estimaba en unos cuatro mil. Los profesores, pues, tenian que ser muchos y, por lo mismo, estaban mal pagados y llegaban mal preparados, porque los estudios de menor categoría robaban alumnos a las institu- 
ciones más solventes. La Compañia de Jesús, que desde 1546 fue apoderándose progresivamente del sistema educativo, otorgaba un buen papel a la formación clásica (hasta el punto de que en ningún otro ambiente se usaron más a menudo las palabras bumanitas y bumanista) y sobre todo a la composición latina. Pero claro está que tales saberes no podían ocupar sino un puesto secundario en la jerarquía de valores de una orden religiosa.

El resultado de la situación, de Carlos V a Felipe IV, fue que la cultura en la línea de los studia bumanitatis se contempló en España con aprecio y respeto, pero nunca se difundió con la misma fortaleza ni impuso el mismo temor reverencial que en otros países. Esa postura equidistante tuvo provechosas consecuencias artísticas.

En especial, la frecuentación de las letras clásicas dejó amplias huellas en la literatura romance: unas huellas que hicieron más notoria la afinidad entre ambas, dando por ende a la más joven una dignidad y un relieve inéditos y refinando el gusto de los nuevos públicos. Pero la literatura vernácula no conoció en la Península una tiranía clasicista tan larga e intensa como en Italia o tan concentrada como en Francia. Por más que admiraran y asimilaran el legado antiguo en muchos aspectos, los grandes escritores españoles no se sintieron obligados a producir una obra destinada sólo a los entendidos, a los connaisseurs o addetti ai lavori, sino que prefirieron dirigirla al común de los lectores o espectadores, para quienes el acatamiento de los modelos clásicos no era en sí mismo ningún valor. En el teatro, Lope de Vega caracterizó lúcidamente tal actitud y la puso en práctica con magnífica eficacia. Pero ni siquiera la complacencia en la erudición que Luis de Góngora despliega en las Soledades y en el Polifemo le impidió ser también el autor de romances y letrillas que andaban en boca de todos. Fue en ese marco de libertad donde se dió la creación del género principal de la modernidad literaria: la novela realista.

Hasta bien entrado el siglo XVII, las máximas novedades de la literatura vulgar fueron en Europa los popularísimos best sellers que llegaban de España: de La Celestina, de Fernando de Rojas, al Quijote, de Miguel de Cervantes, pasando por libros hoy menos recordados, pero en su día incluso más influyentes, como el Marco Aurelio, de Antonio de Guevara, o el Guzmán de Alfarache, de Mateo Alemán. En todos ellos se reconoce un sustrato clásico actuante y a la vez transgredido, con transgresión que constituye su suprema originalidad. La Celestina, así, jamás pierde de vista a Terencio y la comedia humanística, pero rompe todas las jerarquías convencionales dotando de humanidad plena y dimensiones trágicas a unos personajes de ínfima condición social. El Quijote parte de la preceptiva neoaristotélica del Renacimiento tardío para entablar entre la poesía y la historia un dialogo en el curso del cual ambas quedan desbordadas por un nuevo estatuto de la ficción, que marcará el rumbo de toda la novela posterior. Innovaciones tan decisivas como ésas no podían darse en el estricto camino marcado por la tradición más pujante del Humanismo.

Los mejores frutos de los studia bumanitatis se agostan en España a medida que va declinando la casa de Habsburgo. En el siglo siguiente, bajo los Borbones, el lento resurgir del Humanismo en forma de "crítica" o "neoclasicismo" procede ya de otras fuentes. 